\title{
Physique des fluides aux échelles microscopiques pour l'ingénierie des microsystèmes : fabrication et caractérisation
}

Public concerné : étudiants de Master 1

Auteurs : Renaud Dufour ${ }^{\text {a,c,d }}$, Simon Laurette ${ }^{\text {a,b,d }}$, Thomas Dargent ${ }^{\text {a,d }}$, Maxime Harnois ${ }^{\text {a,d }}$, NourEddine Bourzgui ${ }^{\mathrm{a}, \mathrm{d}}$, Vincent Thomy ${ }^{*}, \mathrm{a}, \mathrm{d}$

a) Institut d'Electronique, de Microélectronique et de Nanotechnologie (IEMN) - UMR CNRS

8520, Université Lille 1 Sciences et Technologies, Villeneuve d'Ascq, France

b) Lycée Saint Nicolas, 108 rue de Vaugirard, 75006 PARIS

c) Institut Supérieur de l'Electronique et du Numérique (ISEN) - Lille, France

d) Université Lille I, Villeneuve d'Ascq, France

*E-Mail : vincent.thomy@iemn.univ-lille1.fr

Résumé : Les notions principales rencontrées en microfluidique sont mises en lumières dans ce module. Après une introduction générale sous forme de cours magistraux, les étudiants sont confrontés à des problèmes de dimensionnement de microcanaux à l'aide du logiciel COMSOL Multiphysique®. La suite de l'enseignement est dédiée à la réalisation et à la caractérisation de canaux microfluidiques. La fabrication est effectuée au sein la salle blanche du CNFM de Lille par technique de réplication de polymères. Les étudiants sont également sensibilisés aux questions d'interconnexions des canaux avec les systèmes de contrôle de débit. La dernière partie de ce module consiste enfin à caractériser les systèmes fabriqués et à confronter les résultats expérimentaux aux résultats de simulation.

\section{Introduction}

La microfluidique est un domaine en pleine expansion. Au-delà de la compréhension des phénomènes physiques qui régissent le comportement des fluides à l'échelle micro- voire nanométrique, les champs d'applications sont nombreux et variés : depuis les laboratoires sur puces à la récupération d'énergie en passant par la chimie analytique, la biologie moléculaire et l'industrie pétrolière 1-4. Ces multiples applications expliquent l'engouement actuel pour cette thématique de recherche. Il nous semble donc important de faire découvrir aux étudiants une discipline à laquelle ils pourront être confrontés dans leur avenir professionnel.

Le module mis en place débute par une introduction théorique sous forme de cours magistraux. L'accent est ensuite mis, lors de séances de travaux dirigés (TD), sur la modélisation de phénomènes caractéristiques en microfluidique au moyen du logiciel COMSOL Multiphysique ${ }^{\circledR}$ (modélisation numérique par éléments finis). On aborde ainsi les écoulements laminaires, les phénomènes capillaires, ainsi que le mélange diffusif de deux fluides à l'échelle micrométrique5.

Ces premières séances de TD servent de support pour des travaux pratiques (TP) qui font l'objet de cet article. Lors d'une première séance en salle blanche, un dispositif microfluidique est réalisé puis caractérisé lors d'une seconde séance au sein d'une plateforme dédiée à la recherche. Les étudiants sont alors confrontés à la problématique du mélange en microcanal ainsi qu'aux écoulements diphasiques (liquides non miscibles). Ce module permet une initiation au travail en salle blanche, ainsi qu'à l'utilisation de différents outils de caractérisation (microscopie optique, acquisition vidéo, utilisation de contrôleurs de débit, etc.). Les notions théoriques acquises au préalable durant les cours et TD de simulation permettent aux étudiants d'avoir les bases suffisantes pour appréhender leurs résultats expérimentaux et les confronter à des modèles théoriques. 


\section{Réalisation technologique}

Le travail de microfabrication se déroule dans la salle blanche du Pôle CNFM de Lille dédiée à l'enseignement. Cette séance de quatre heures a pour but de sensibiliser les étudiants à l'une des méthodes de microfabrication les plus couramment utilisées dans le domaine de la microfluidique. Cette méthode, dite de lithographie douce, consiste à créer des microcanaux via la réplication d'un élastomère sur un moule réalisé en silicium (Figure 1).

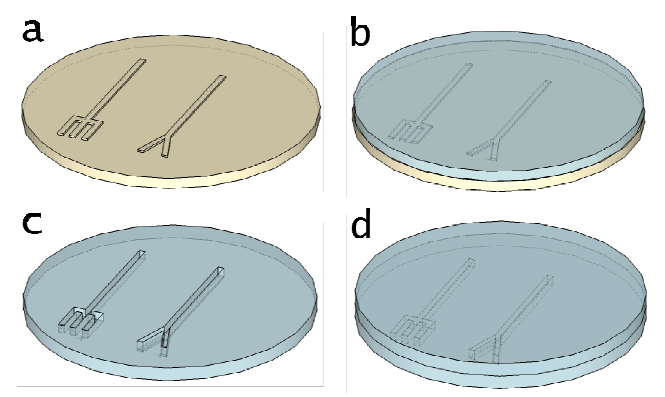

Figure 1: Méthode de fabrication par lithographie douce : (a) On utilise un moule de silicium obtenu via des techniques de microfabrication conventionelles (photolithographie, gravure profonde). (b) L'élastomère est coulé dans ce moule puis réticulé. (c) Après démoulage, on obtient la réplique des microcanaux dans l'élastomère. (d) Les canaux sont fermés via le collage avec un second élément d'élastomère (capôt), il reste ensuite à découper le dispositif et à assembler les capillaires d'entrées et sorties (cf. Figure 2).

Durant la séance, les étudiants abordent les aspects suivants :

- La fabrication d'un moule en silicium via des techniques de microfabrication conventionnelles utilisées dans l'industrie de la microélectronique (lithographie optique et gravure profonde sèche).

- La préparation et réplication de l'élastomère: on utilise le polydimethylsiloxane (PDMS), matériau le plus utilisé pour le prototypage rapide de dispositifs microfluidiques.

- L'assemblage du dispositif, qui inclue le collage des différents éléments en PDMS (canaux microfluidiques et capôt), ainsi que la fixation des capillaires d'entrées et de sorties.

Ces différentes étapes sont détaillées dans les sections suivantes.

\section{1) Fabrication du moule silicium (effectué par l'encadrant au préalable)}

Le moule de silicium, servant de base au procédé de lithographie douce, est fabriqué au préalable par l'encadrant. La méthode de fabrication est expliquée aux étudiants en début de séance (environ 15 minutes).

Le moule est obtenu à partir d'un substrat 3 pouces de silicium. On effectue dans un premier temps une étape de lithographie optique à l'aide d'une résine positive (Megaposit SPR220, épaisseur de $7.5 \mu \mathrm{m}$ ). Les canaux microfluidiques sont ensuite obtenus par gravure sèche profonde $\mathrm{du}$ silicium. Suite à la gravure, une couche de fluoro-polymère hydrophobe (C4F8, $20 \mathrm{~nm})$ est déposée sur le moule afin de faciliter l'étape de démoulage de l'élastomère.

Deux géométries de microcanaux sont ainsi réalisées. La première consiste en une jonction « $\mathrm{Y} »$, la seconde en une jonction en croix (Figure 1-a). Ces géométries de base permettent d'étudier les principaux phénomènes caractéristiques en microfluidique tels que le mélange diffusif (jonction « $\mathrm{Y} »$, cf. section III-1), et la formation de trains de goutte (jonction en croix, cf. section III-2). La largeur des canaux est de $100 \mu \mathrm{m}$, leur profondeur de $180 \mu \mathrm{m}$ et leur longueur de $1 \mathrm{~cm}$.

\section{2) Préparation et réplication du PDMS (environ 1 heure)}

Le PDMS est obtenu en mélangeant un pré-polymère (Sylgard 184 Silicon Elastomer) avec un agent réticulant (Sylgard 184 curing agent) à un ratio massique de 10:1. Une étape de dégazage sous vide est nécessaire afin d'éliminer les bulles formées durant le mélange. 
Une partie du mélange obtenu est versée sur le moule (Figure 1-b), une autre partie est versée dans une boîte de pétri afin d'obtenir une surface plane servant de capôt au dispositif. Les deux éléments sont ensuite recuits à $110^{\circ} \mathrm{C}$ pendant 10 minutes sur plaque chauffante puis démoulés manuellement.

\section{3) Assemblage du dispositif (environ 1 heure)}

La première partie de l'assemblage consiste à coller les deux éléments réalisés en PDMS (microcanaux et capôt). Pour cela, les deux surfaces sont exposées à un plasma O2 (Bâti PICO, Diener electronic, paramètres : $50 \mathrm{~W}, 150 \mathrm{mT}, 45 \mathrm{~s}$ ). Cela a pour but de modifier la chimie de surface du PDMS en produisant des terminaisons silanol (Si-OH) rendant le PDMS temporairement hydrophile. La mise en contact des deux surfaces de PDMS permet alors leur collage (Figure 1-d).

La seconde partie de l'assemblage consiste à découper les échantillons au scalpel, puis à insérer les capillaires (Polymicro technologies ref: TSP100170) dans les microcanaux pour les coller (colle Araldite $\left.{ }^{\circledR}\right)$. On obtient alors des microsystèmes fluidiques tel que représentés sur la Figure 2-a. Les autres extrémités des capillaires sont reliées à des seringues en plastique par l'intermédiaire de connectiques spécifiques de type «luer lock» (Figure 2-b). La séance se termine par un test des microsystèmes qui consiste à injecter de l'eau dé-ionisée colorée afin de vérifier la bonne fixation des capillaires et l'absence de fuites entre les deux éléments de PDMS.

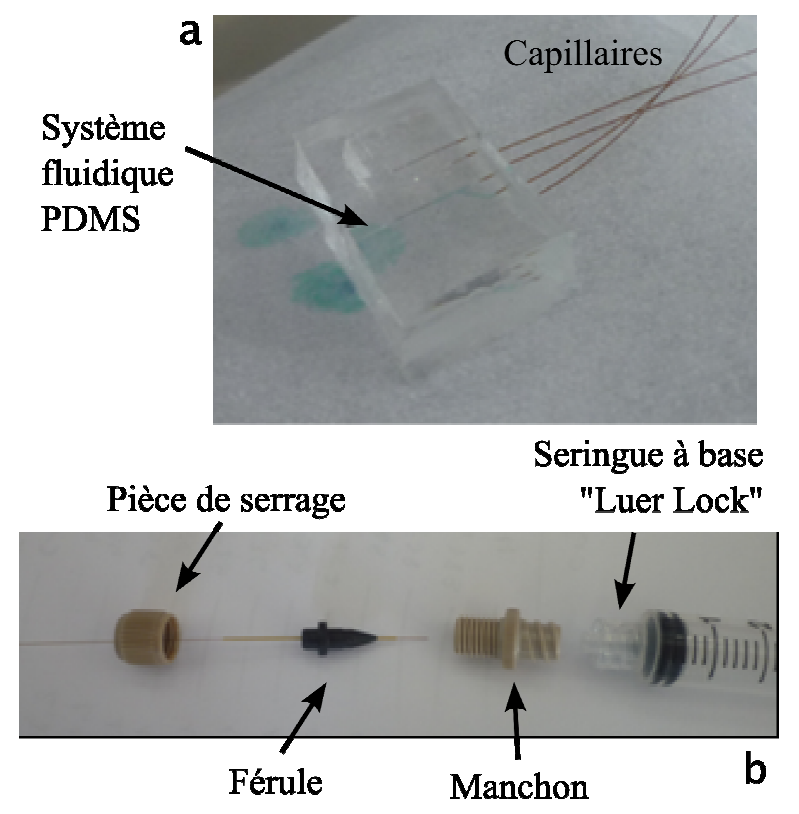

Figure 2 : (a) Dispositif microfluidique en PDMS après découpe au scalpel et fixation des capillaires. (b) Connexion d'un capillaire à une seringue en plastique via une connectique « luer lock ".

\section{4) Caractérisation microfluidique}

Cette seconde séance de quatre heures se déroule au sein de la plateforme de caractérisation microfluidique de l'IEMN, permettant ainsi aux étudiants de côtoyer au plus près le monde de la recherche.

Au début de la séance (30 minutes environ), les étudiants sont initiés à l'utilisation des principaux instruments utilisés dans le laboratoire, à savoir :

- les microscopes optiques classique et en fluorescence, permettant de visualiser les écoulements au sein des microcanaux,

- les caméras, logiciels d'acquisition et réglages spécifiques (rapidité, sensibilité, profondeur de champ, etc.), 
- les systèmes de contrôle permettant d'injecter des liquides dans les systèmes microfluidiques (contrôleurs en débit ou en pression), avec l'explication des avantages et inconvénients de chacun.

Lors de ce TP, nous avons opté pour des pousses seringues (contrôle en débit) de par la simplicité de mise en œuvre de l'expérience. Afin d'illustrer un des problèmes de la manipulation de liquides en microcanaux par des pousse seringues, le premier point abordé par les étudiants porte sur le temps de latence de l'écoulement après avoir stoppé le débit. Les étudiants se rendent compte que plusieurs minutes s'écoulent avant l'arrêt total du flux du liquide. Ceci peut s'expliquer entre autres par la déformation de la seringue en plastique soumise à la pression du pousse-seringue ainsi qu'à la résistance hydrodynamique du système.

Les étudiants réalisent ensuite deux séries d'expériences sur le banc expérimental représenté sur la figure 2. Ce banc comprend : un microscope optique classique muni d'une caméra CCD standard, deux puis trois contrôleurs de débit (pousse-seringues) et un ordinateur permettant la commande des pousses seringue via une interface Labview et l'acquisition vidéo. On alloue environ 1h30 par expérience (rédaction d'un rapport compris).

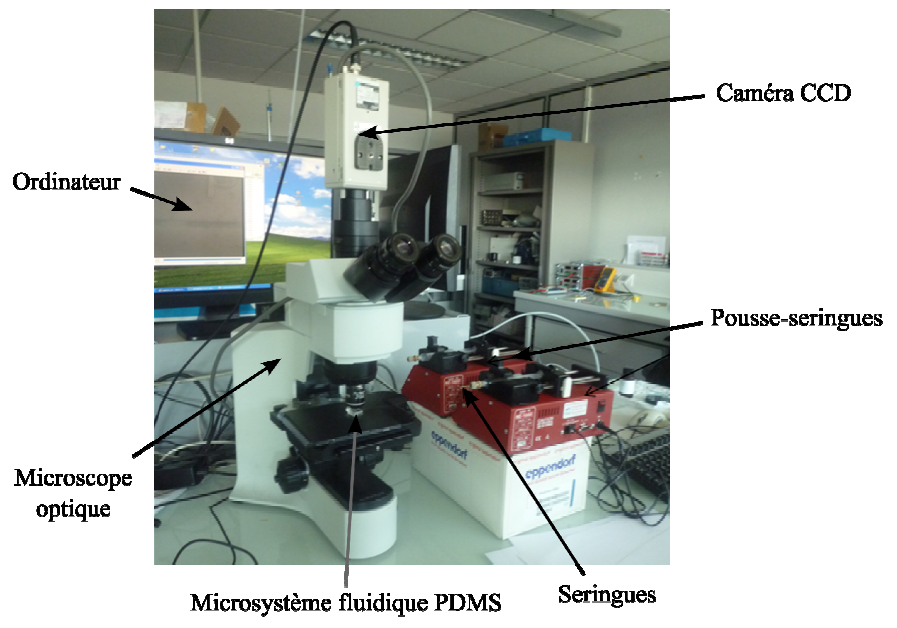

Figure 3 : Photographie du banc expérimental comprenant un ordinateur de contrôle, une caméra CCD (Hamamatsu C11440) connectée à un microscope optique (Olympus BX41M), et trois pousse-seringues (New Era NE-1000).

A travers deux expériences complémentaires, les étudiants sont amenés à réfléchir sur une situation couramment observée en microfluidique : la rencontre de deux solutions distinctes lors d'une jonction. Selon la miscibilité ou non des deux solutions, les effets observés diffèrent largement : c'est ce que mettent en évidence les paragraphes suivants.

\section{5) Première expérience : Diffusion de deux liquides miscibles}

Aux dimensions macroscopiques, le mélange de deux solutions miscibles est quasi-instantané et est engendré par des flux convectifs turbulents. Dans les écoulements microfluidiques, le nombre de Reynolds est en général inférieur ou proche de 1 et mène à un écoulement laminaire où l'effet de la viscosité est prédominant. Le mélange ne peut donc s'effectuer par convection et c'est la diffusion (mouvement brownien) des espèces qui permet le mélange. Celle-ci est caractérisée par un coefficient $\mathrm{D}$, associé à un temps de diffusion tdiff sur une longueur caractéristique $1: \boldsymbol{t}_{\text {diff }}=\boldsymbol{l}^{\mathbf{2}} / \boldsymbol{D}$. Pour que le mélange ait lieu dans les microcanaux, il faut que ce temps de diffusion soit inférieur au temps de séjour $t_{\text {sejour }}$ des solutions dans la zone de mélange. On définit alors le nombre de Péclet $P_{e}$ comme le rapport $t_{\text {diff }} / t_{\text {sejour }}$, soit $P_{e}=l . v / D, v$ étant la vitesse caractéristique de l'écoulement. On comprend alors que $P_{e}<<1$ correspond à une situation de mélange et $P_{e} \gg 1$ à une situation où le mélange n'a pas eu le temps de s'effectuer. 
Cette discussion théorique a été menée avec les étudiants lors des séances de TD sous COMSOL où ils ont pu visualiser l'effet du nombre de Péclet sur le mélange. Un exemple extrait d'une simulation COMSOL est représenté sur la Figure 4 (reproduit à partir de la référence 5). On se propose ici d'en effectuer une validation expérimentale.

A cette fin, on injecte dans une jonction Y de l'eau dé-ionisée (EDI), et de l'encre. Le coefficient de diffusion de l'encre dans l'eau peut être estimé à $D \approx 10^{-5} \mathrm{~cm}^{2} \cdot \mathrm{s}^{-1}$. On se place dans le cas où les deux solutions sont injectées avec le même débit $Q$. A partir des dimensions des microcanaux fabriqués, on demande aux étudiants de déterminer les débits d'injection permettant d'obtenir : (1) le mélange des deux solutions, ou (2) aucun mélange entre les deux solutions. Ceci leur permet (a) de ré-investir la notion de nombre de Péclet vue en TD, (b) de manipuler les formules de base reliant débit et vitesse, et également (c) d'adopter une démarche d'investigation. Ils sont ensuite amenés à vérifier expérimentalement les valeurs de débit proposées. Grâce à l'encre, on observe en effet immédiatement (après le régime transitoire lié à la mise en mouvement du fluide et à la compression de la seringue) si le mélange a eu lieu ou non.

Enfin, cette étude permet d'introduire la nécessité de micromélangeurs pour les applications lab-on-chip.

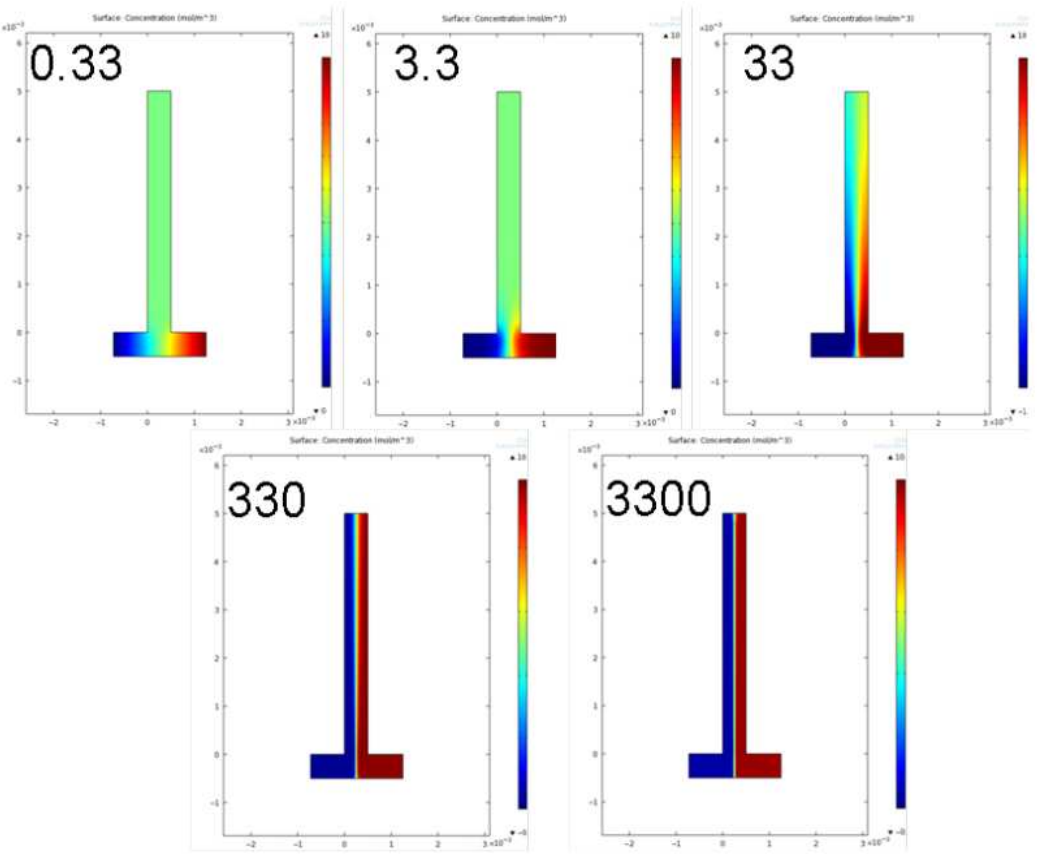

Figure 4 : Evolution du mélange dans un microcanal en fonction du nombre de Péclet. Les deux liquides injectés dans la jonction sont représentés en rouge et bleu. Pour un nombre de Péclet faible $(P e=0.33$ ou 0.3 ), les deux liquides sont mélangés à la sortie du microcanal. Lorsque le nombre de Péclet augmente, le mélange n'a plus le temps de s'effectuercar le temps nécessaire à la diffusion du liquide devient plus important que le temps de séjour dans le canal.

6) Seconde expérience : Génération de trains de gouttes avec deux liquides non-miscibles La seconde expérience concerne l'écoulement de deux liquides non-miscibles. Dans ce cas, on observe non plus un phénomène de diffusion mais la formation d'un écoulement diphasique. La complexité de ce phénomène empêche une analyse simple en TD de simulation COMSOL. En revanche, l'approche expérimentale choisie ici permet une bonne appréhension des phénomènes rencontrés. On utilise la méthode dite de «flow-focusing » afin de créer des trains de gouttes dans un microcanal ${ }^{7,8}$. Cette méthode consiste à utiliser une jonction en croix telle que représentée sur la Figure 5. Les liquides utilisés sont de l'eau déionisée (colorée avec un colorant alimentaire) et de 
l'huile. L'eau est injectée à un débit $Q_{e a u}$ via l'entrée $a$, et l'huile à un débit $Q_{\text {huile }}$ via les entrées $b$ et $c$. Les deux flux d'huile viennent «pincer» le flux d'eau, ce qui crée un écoulement diphasique dans le canal de sortie (au niveau de la jonction, il apparait une compétition entre la force de tension interfaciale, tendant à retenir la goutte d'eau dans le canal $a$ ) et la pression de l'huile tendant à détacher la goutte). La longueur $L$ des gouttes d'eau peut alors être modulée via le rapport des deux débits $Q_{\text {huile }} / Q_{\text {eau }}$.
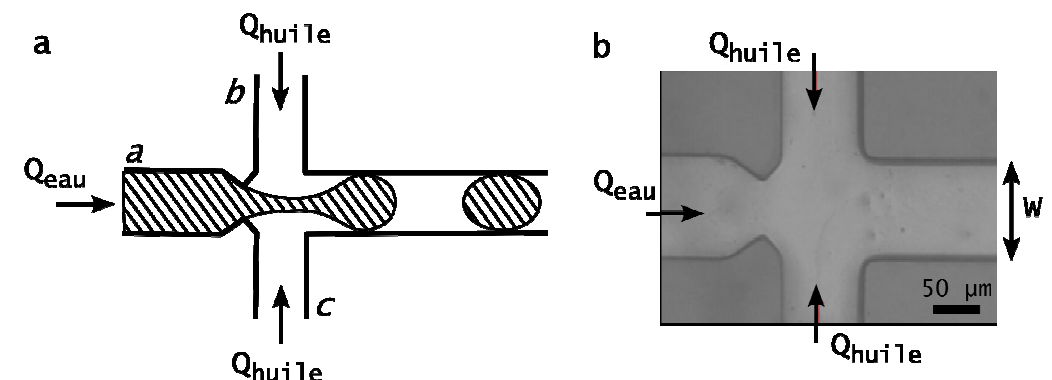

Figure 5 : (a) Principe du " flow-focusing ", L'eau est injectée via l'entrée a, et l'huile via les entrées b et c. La restriction du canal a à l'entrée de la jonction en croix permet de faciliter le pincement de l'eau par l'huile afin d'obtenir un écoulement diphasique (gouttes d'eau dans l'huile). (b) Microcanaux réalisés.

D'un point de vue théorique, le rapport entre la longueur des gouttes $L$ et la largeur du canal $W$ est donné par l'équation (1). Dans cette équation, $\alpha$ est un paramètre qui dépend de la géométrie de la jonction en croix.

$$
\frac{L}{W}=1+\alpha \frac{Q_{\text {eau }}}{Q_{\text {huile }}}
$$

Afin d'étudier ce phénomène, les étudiants sont invités à faire varier le débit d'huile, à débit d'eau constant. Pour chaque rapport $Q_{\text {eau }} /$ Qhuile, une acquisition vidéo est effectuée, à partir de laquelle les étudiants mesurent la longueur L des gouttes d'eau obtenues. Un exemple est représenté sur la Figure 6.
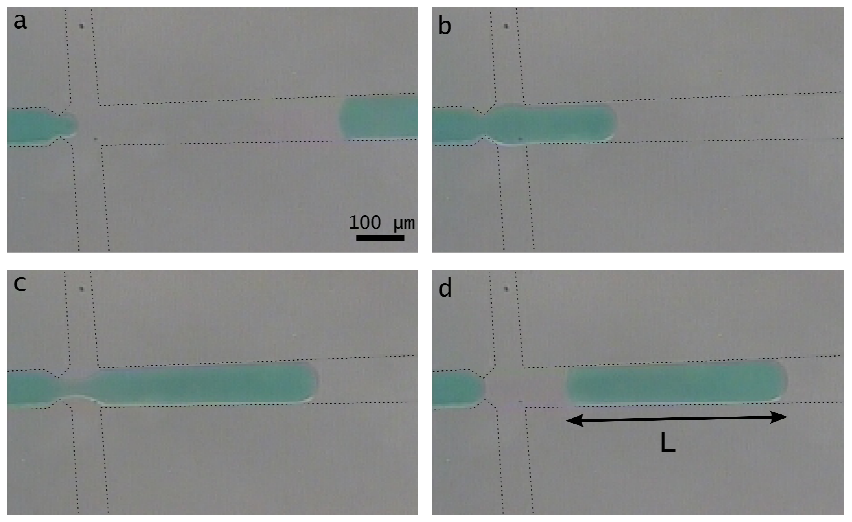

Figure 6 : Génération de gouttes d'eau dans l'huile par la méthode de flow-focusing.

Les débits d'eau et d'huile sont respectivement Qeau $=5 \mu \mathrm{L}$ et Qhuile $=40 \mu \mathrm{L}$. Soit Qeau/Qhuile $=0.125$. On obtient ici Un rapport $\mathrm{L} / \mathrm{W}=4.7$.

On demande ensuite de représenter graphiquement l'évolution de L/W en fonction de $\mathrm{Q}_{\text {eau }}$ / $Q_{\text {huile }}$ et d'en extraire le paramètre $\alpha$. Une synthèse des résultats est représentée sur la Figure 1, on obtient ici $\alpha \approx 30$. 


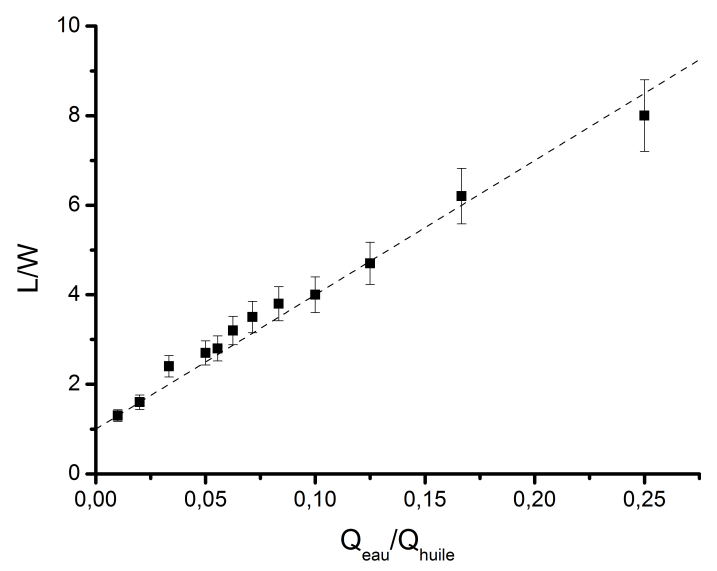

Figure 7 : Résultats obtenus pour la génération de gouttes d'eau dans l'huile via la méthode de flow focusing. On obtient ici $L / W=1+30 \cdot Q_{\text {eau }} / Q_{\text {huile }}$.

\section{Conclusion}

Ces travaux pratiques permettent aux étudiants, lors d'une première séance, de s'initier au travail en salle blanche via la fabrication et l'assemblage d'un microsystème fluidique. La seconde séance leur permet de découvrir l'environnement d'un laboratoire de recherche, d'apprendre à utiliser différents instruments de caractérisation, et de confronter leurs résultats expérimentaux à des notions théoriques acquises lors des cours magistraux et TD. A la frontière de la thématique EEA, ce module a pour objectif de sensibiliser les étudiants à une approche pluridisciplinaire des sciences pour l'ingénieur. Cette formation est également ouverte à d'autres étudiants de différents parcours (Master 2 Biophysique, Biomédicale ainsi que 4éme année d'école d'ingénieur). Quelques soient les profils des étudiants, les retours ont été extrêmement positifs en terme d'implications et de compréhension.

\section{Références}

1. Whitesides G. M. The origins and the future of microfluidics. Nature 442, 368-373 (2006).

2. Colin A., Squires T. M. \& Bocquet L. Soft matter principles of microfluidics. Soft Matter $\mathbf{8}$, 10527-10529 (2012).

3. Squires T. M. \& Quake S. R. Microfluidics: Fluid physics at the nanoliter scale. Reviews of Modern Physics 77, 977-1026 (2005).

4. Stone H. A., Stroock A. D. \& Ajdari, A. Engineering Flows in Small Devices. Annual Review of Fluid Mechanics 36, 381-411 (2004).

5. Laurette S., Dufour R., Brendriaa F., Thomy V. \& Bourzgui, N.-E. Physique des fluides aux échelles microscopiques pour l'ingénierie des microsystèmes : l'apprentissage par la simulation. J3eA 10, 0001 (2011).

6. Patrick Tabeling Introduction à la microfluidique. 254 (2003).

7. Anna, S. L., Bontoux, N. \& Stone, H. A. Formation of dispersions using "flow focusing" in microchannels. Applied Physics Letters 82, 364-366 (2003).

8. Garstecki P., Fuerstman M. J., Stone H. A. \& Whitesides G. M. Formation of droplets and bubbles in a microfluidic T-junction-scaling and mechanism of break-up. Lab on a Chip 6, 437446 (2006). 